\title{
Elastic constants of the hard-sphere glass: a density functional approach
}

\author{
Hartmut Löwen† \\ Laboratoire de Physique, Ecole Normale Supérieure de Lyon, 69364 Lyon Cédex 07, \\ France
}

\begin{abstract}
The free energy and the static elastic constants of a hard-sphere glass are calculated using density functional theory. More specifically, Bennett's glass model is used where the packing fraction of random closed packing, $\eta_{\mathrm{RCP}}$, is taken as an adjustable parameter. As regards the density functional, the modified weighted density approximation (MWDA) is employed. The results are compared with the FCC hard-sphere crystal. It is found that the free energy of the glass is always higher than the crystal free energy; however, for high densitjes and high $\eta_{R C P}$, it is lower than the free energy of a fluid resulting in a first-order liquid-to-glass phase transition. For the same temperature and densities, the bulk modulus in the glass is higher than that in the FCC crystal. For high $\eta_{\mathrm{RCP}}$ and high densities, the shear modulus is smaller than that of a polycrystalline sample.
\end{abstract}

\section{Introduction}

The freezing mechanism of a supercooled hard-sphere fluid into a glass is a fascinating subject and, in order to understand it theoretically, several methods have been used: computer simulation (see e.g. [1]), density functional [2,3] and mode coupling theories (see e.g. [4]). As regards density functional theories, which were originally developed [5] to describe freezing into a regular solid lattice, the first attempt to calculate the limit of stability of a fluid with respect to the glass was made in 1985 by Singh, Stoessel and Wolynes [2]. This was then reconsidered by Baus and Colot [3] within an improved density functional. In particular, Baus and Colot used Bennett's [6] dense random packing model of hard spheres to describe the structure and especially the site-site correlation function of the glass. Here, by simple scaling, the packing fraction of random closed packing, $\eta_{\mathrm{RCP}}$, was used as an additional parameter of the theory. It was found that the glass is stable with respect to the fluid for $\eta_{\mathrm{RCP}}>0.69$, but remains metastable with respect to the FCC crystal.

In the last few years, considerable success in the description of the hard-sphere crystal was made with socalled weighted density approximations for the density functional $[7,8,9,10,11]$, see [12] for a recent review and a compilation of the data. It is the aim of the present paper to apply the weighted density functional technique to Bennett's hard-sphere glass and to calculate the static elastic constants of the glass as a function of $\eta_{R C P}$ and density. This can be done along similar lines as the calculation of the elastic constants in the FCC hard-sphere crystal $[13,14]$. It turns out that the bulk modulus $E$ of the glass is higher than that of the FCC crystal but lower than

† Permanent address: Sektion Physik der Universität München, 8000 München 2, Federal Republic of Germany. 
that of a liquid. The shear modulus $G$, on the other hand, is smaller than that of a polycrystalline sample if the density and $\eta_{\mathbf{R C P}}$ are high. As is well known, concerning elastic response the glass can be viewed as something intermediate between a crystal and a liquid.

It is interesting to compare this result with simulations of a simple amorphous binary alloy near the glass transition by Barrat et al [15]. They also found that the bulk modulus of the glass lies between fluid and solid, the shear modulus being smaller than that of a polycrystalline sample for any density or temperature, respectively.

\section{Density functional theory}

Several different versions of weighted density techniques have been proposed. We choose the most simple one of Denton and Ashcroft [9], called modified weighted density approximation (MWDA) which is optimal in an expansion of a coarse grained reference functional up to second order [16].

Let us first outline how to apply the MWDA to the hard-sphere glass: The local density of the glass, $\rho(\boldsymbol{r})$, is assumed to be a sum of isotropic Gaussian peaks centred around a set of prescribed sites $\left\{R_{j}\right\}$

$$
\begin{aligned}
& \rho(\boldsymbol{r})=\sum_{j=1}^{N} \phi\left(\boldsymbol{r}-\boldsymbol{R}_{j}\right) \\
& \phi(\boldsymbol{r})=\left(\frac{\alpha}{\pi}\right)^{3 / 2} \exp \left(-\alpha r^{2}\right)
\end{aligned}
$$

The sites $\left\{\boldsymbol{R}_{j}\right\}$ are distributed randomly, e.g. according to Bennett's cluster growth law, and $\alpha$ is the single variational parameter. The free energy density functional for hard spheres, $F[\rho]$, can be split into an ideal part, $F_{\text {id }}[\rho]$, and an excess term, $F_{\text {ex }}[\rho]$, where

$\beta F_{\text {id }}[\rho]=\int \mathrm{d}^{3} r \rho(\boldsymbol{r})\left[\ln \left(\Lambda^{3} \rho(\boldsymbol{r})\right)-1\right] \approx N\left\{-\frac{5}{2}+\ln \left[\Lambda^{3}\left(\frac{\alpha}{\pi}\right)^{3 / 2}\right]\right\}$

for large $\alpha, \beta=1 / k_{\mathrm{B}} T$ being the inverse temperature and $\Lambda$ the thermal wavelength. Within the MWDA, $F_{\text {ex }}[\rho]$ is given by

$$
F_{\text {ex }}[\rho]=N \Psi(\hat{\rho})
$$

where $\Psi$ is the excess free energy per particle in a homogeneous system. The weighted density $\hat{\rho}$ is given by

$$
\hat{\rho}=\frac{1}{N} \int \mathrm{d}^{3} r \int \mathrm{d}^{3} r^{\prime} \rho(\boldsymbol{r}) \rho\left(\boldsymbol{r}^{\prime}\right) w\left(\left|\boldsymbol{r}-\boldsymbol{r}^{\prime}\right| ; \hat{\rho}\right)
$$

Here, the weight function is determined by the condition that the Ornstein-Zernike direct correlation function of the homogeneous system, $c(r ; \rho)$, is reproduced exactly. This yields

$$
w(r ; \rho)=-\frac{1}{2 \Psi^{\prime}(\rho)}\left[\frac{1}{\beta} c(r ; \rho)+\frac{1}{V} \rho \Psi^{\prime \prime}(\rho)\right]
$$


where $V$ denotes the volume of the system and the primes derivatives with respect to $\rho$. Inserting (4), (1a) and (1b) into (3) yields the fundamental implicit equation for $\hat{\rho}$ :

$$
\begin{gathered}
2 \Psi^{\prime}(\hat{\rho}) \hat{\rho}=-\rho \hat{\rho} \Psi^{\prime \prime}(\hat{\rho})-\int \mathrm{d}^{3} r \int \mathrm{d}^{3} \boldsymbol{r}^{\prime} \int \mathrm{d}^{3} R c\left(\left|\boldsymbol{r}-\boldsymbol{r}^{\prime}\right| ; \hat{\rho}\right) \\
\times \phi(\boldsymbol{r}-\boldsymbol{R}) \phi\left(\boldsymbol{r}^{\prime}\right)\left[\delta(\boldsymbol{R})+\rho g_{L}(\boldsymbol{R})\right]
\end{gathered}
$$

where $\rho=N / V$ is the mean density. The essential input, characterizing the glassy state, is the site-site correlation function $g_{L}(\boldsymbol{R})$. Following Baus and Colot [3], we choose

$$
g_{L}(\boldsymbol{R})=g_{\mathrm{B}}\left[\left(\frac{\eta}{\eta_{\mathrm{RCP}}}\right)^{1 / 3} R\right]
$$

where $\eta$ denotes the packing fraction, $\eta=\frac{1}{6} \pi \rho \sigma^{3}$, ( $\sigma$ being the hard-sphere diameter $)$ and $g_{\mathrm{B}}(R)$ is Bennett's site-site correlation function.

Before discussing the results, two remarks are in order: First, one should note that the $\eta_{\mathrm{RCP}}$-dependence in (6) is obtained by simple scaling. This should be a good approximation for $0.6<\eta_{\mathbf{R C P}}<0.66$. Higher $\eta_{\mathrm{RCP}}$ are relevant if one considers finite clusters [3]. In this case it is doubtful whether the $\eta_{\mathrm{RCP}}$-dependence of $g_{L}(\boldsymbol{R})$ is still given by (6). Nevertheless, let us take (6) as an approximation in order to have an easily comprehensible model for the glass where $g_{L}(\boldsymbol{R})$ is known explicitly. Furthermore, the results can then directly be compared with that of [3]. For a more realistic $g_{L}(\boldsymbol{R})$ the free energy of the glass increases.

The second remark is more general concerning the description of a glassy state by the function $g_{L}(\boldsymbol{R})$ within density functional theory. This is only possible, if the densities are coupled quadratically in the functional which is the case in the MWDA [9] but not in the original weighted density approximations $[7,8]$.

The general steps are now clear. The free energy of a Bennett glass, characterized by $\eta_{\mathrm{RCP}}$ and mean density $\rho$, can be calculated by minimizing $F[\rho]$ with respect to $\alpha$. For each minimization step, the implicit equation (6) for $\hat{\rho}$ has to be solved. Throughout this paper, we use the Percus-Yevick expression for $c(r ; \hat{\rho})$ and $\Psi(\hat{\rho})$. This is justified since $\hat{\rho}$ turns out to be considerably lower than $\rho$.

The result for the free energy is displayed in figure 1 . The free energy of the glass is always higher than the free energy of the FCC crystal. But the glass can become stable with respect to the fluid for high densities and high $\eta_{\mathrm{RCP}}$. This is accompagnied by a first-order phase transition which can be calculated using the familiar double-tangent construction. Within the MWDA, a fluid-glass transition takes place if $\eta_{\mathrm{RCP}}>\eta_{\mathrm{RCP}}^{0}=0.672$. At this stage a remark is in order (see also the discussion at the end of section 3): Since, within the density functional approach, merely free energies are compared, one does not obtain information on the dynamics of the phase transition. For the glass transition dynamic diagnostics are important, which, however, are missing in our approach.

Baus and Colot set $g_{\mathrm{B}}(R)=0$ for $1<R / \sigma<1.025$ and obtained $\eta_{\mathrm{RCP}}^{0}=0.69$. In the MWDA the free energies of the glass are reduced further if this modified $g_{\mathrm{B}}(r)$ is used. In the considered $\rho$ and $\eta_{\mathrm{RCP}}$ range, the differences are between $2 \%$ and $12 \%$ and $\eta_{\mathrm{RCP}}^{0}=0.668$. As is also known from the hard-sphere crystal [17], the density functional of Baus and Colot yields coexisting densities that are too high. 


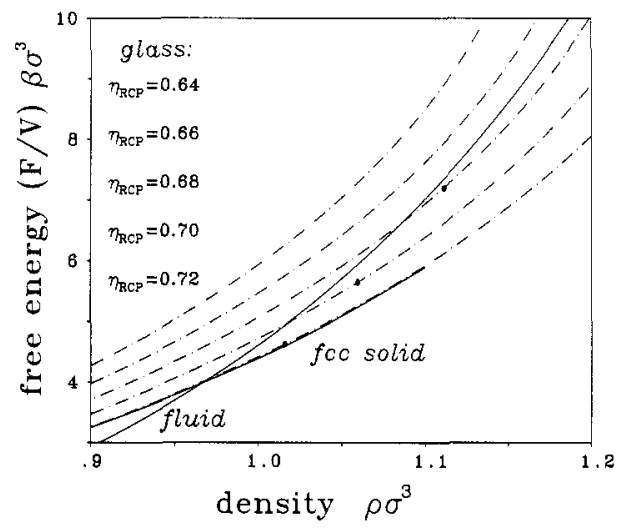

Figure 1. Helmholtz free energy of a Bennett hard-sphere glass versus density for five different random close packing fractions $\eta_{\mathrm{RCP}}(-\ldots-), F$ decreases for increasing $\eta_{\mathrm{RCP}}$. We choose the thermal wavelength to be $\Lambda \equiv \sigma$. For comparison, the corresponding fluid and FCC solid values are also shown (from [9]). The glass remains metastable with respect to the crystal but becomes stable with respect to the fluid if $\eta_{R C P}>0.671$. $\bullet$ glass density, $\rho^{g l}$, coexisting with the fluid.

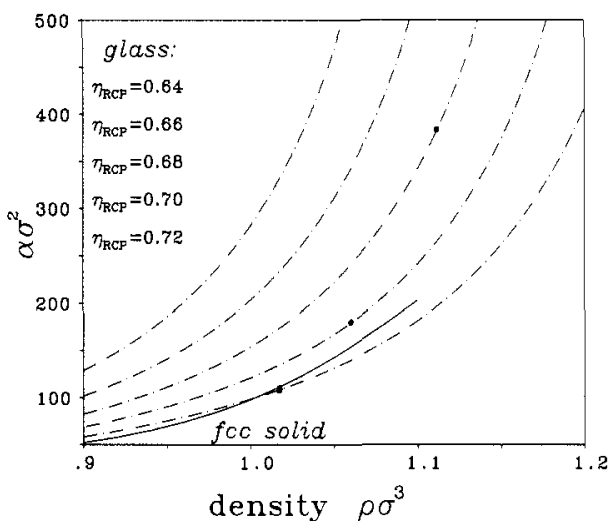

Figure 2. Localization parameter, $\alpha$, versus density for the hard-sphere glass. For comparison, the data for the FCC crystal from [9] are also shown. • coexisting glass density $\rho^{\mathrm{gl}}$.

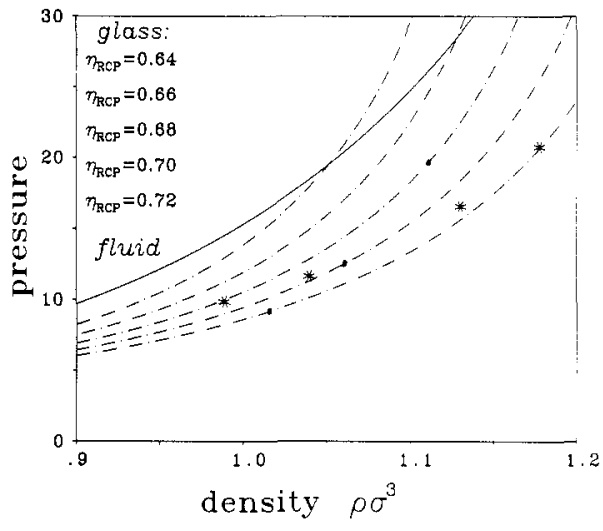

Figure 3. Pressure (in units of $\left(\beta \sigma^{3}\right)^{-1}$ ) versus density for the hard-sphere glass. _-, liquid data from the Carnahan-Starling equation of state. *, hard-sphere FCC crystal, from [22]; $\bullet$, coexisting glass density $\rho^{\mathrm{gl}}$.

The results for the localization parameter $\alpha$, used as variational parameter, and the pressure $p$, obtained by differentiating the free energy per volume, $f=F / V$, as

$$
p=\rho \frac{\partial f}{\partial \rho}-f
$$

are shown in figures 2 and 3 , respectively. In analogy with the solid one can define a Lindemann parameter $L$ as the ratio of the mean square displacements of the atoms and the mean interparticle spacing

$$
L=\left(\frac{3}{2 \alpha d^{2}}\right)^{1 / 2}
$$


with

$$
d=\sigma\left(\frac{\eta_{\mathrm{RCP}}}{\eta}\right)^{1 / 3}
$$

It turns out that for a glass $L$ is not very different from the crystal value. This is consistent with the molecular dynamics simulation of a soft-sphere binary alloy by Roux et al [18]. Explicit results for the coexisting fluid/glass data are summarized in table 1 .

Table 1. Fluid-glass coexistence data for three different random close packing fractions, $\eta_{\mathrm{RCP}} . \rho^{\mathrm{fl}}$ and $\rho^{\mathrm{gl}}$ are the coexisting fluid and glass densities, respectively, $p$ is the corresponding pressure, $\alpha$ the localization parameter and $L$ the Lindemann parameter of the hard-sphere glass. The units of $\rho, p$ and $L$ are $\sigma^{-3},\left(\beta \sigma^{3}\right)^{-1}$ and $\sigma^{-2}$, respectively.

\begin{tabular}{lllrrl}
\hline$\eta_{\mathrm{RCP}}$ & $\rho^{\text {fl }}$ & $\rho^{\mathrm{g}^{1}}$ & \multicolumn{1}{l}{$p$} & \multicolumn{1}{l}{$\alpha$} & $L$ \\
\hline 0.68 & 1.055 & 1.111 & 19.9 & 362 & 0.094 \\
0.70 & 0.963 & 1.060 & 12.9 & 161 & 0.14 \\
0.72 & 0.899 & 1.016 & 9.6 & 87 & 0.18 \\
\hline
\end{tabular}

It would be interesting also to test other density functional approaches (e.g. the recently proposed form of [10]) and to compare with [3] and our results.

\section{Elastic constants}

Let us now turn to the elastic properties of the glass. For a crystal with cubic symmetry, three independent elastic constants $C_{11}, C_{12}, C_{44}$ are present (throughout the paper the notation of $\mathrm{Xu}$ and Baus [14] is used). Alternatively, one can also introduce the bulk modulus $E$ and two shear moduli $G$ and $G^{\prime}$ as independent constants, see [15]. They are related to $C_{11}, C_{12}, C_{44}$ by $E=\frac{1}{3}\left(C_{11}+2 C_{12}\right), G=C_{44}, G^{\prime}=\frac{1}{2}\left(C_{11}-C_{12}\right)$. In our case, however, the glass represents an isotropic solid, hence only two independent elastic constants exist. The two shear moduli are equal in this case, $G=G^{\prime}$.

The bulk modulus can simply be obtained by differentiating the free energy

$$
E=\rho^{2} \partial^{2} f / \partial \rho^{2}
$$

where $f=F / V$. The results are shown in figure 4 .

The calculation of $G$ is more sophisticated. We perform an uniaxial stretch of the glass along the $x$ axis. This means that the site-site correlation function $g_{L}(R)$ is now chosen to be

$$
g_{\mathrm{B}}\left\{\left(\frac{\eta}{\eta_{\mathrm{RCP}}}\right)^{1 / 3}\left[\left(\frac{X}{1-\epsilon}\right)^{2}+Y^{2}+Z^{2}\right]^{1 / 2}\right\}
$$

where $\epsilon$ is a smallness parameter and $R=(X, Y, Z) . C_{11}$ is then given [14] by

$$
\left.\frac{1}{V} \frac{\partial^{2} F}{\partial \epsilon^{2}}\right|_{\epsilon=0}
$$




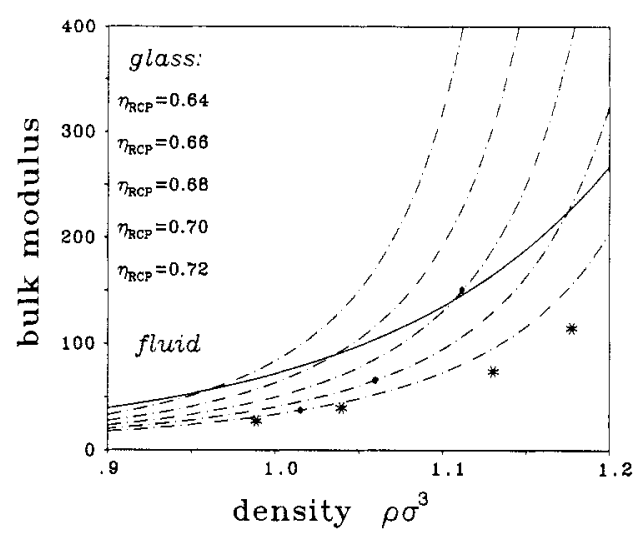

Figure 4. Bulk modulus $E$ (in units of $\left(\beta \sigma^{3}\right)^{-1}$ ) versus density for the hard-sphere glass for different $\eta_{\mathrm{RCP}}$. $E$ decreases for increasing $\eta_{\mathrm{RCP}}$. The corresponding fluid data, obtained from the Carnahan-Starling equation of state, FCC solid data (*) (from [22]) and coexisting glass density $\rho^{\mathrm{gl}}(\bullet)$ are also shown.

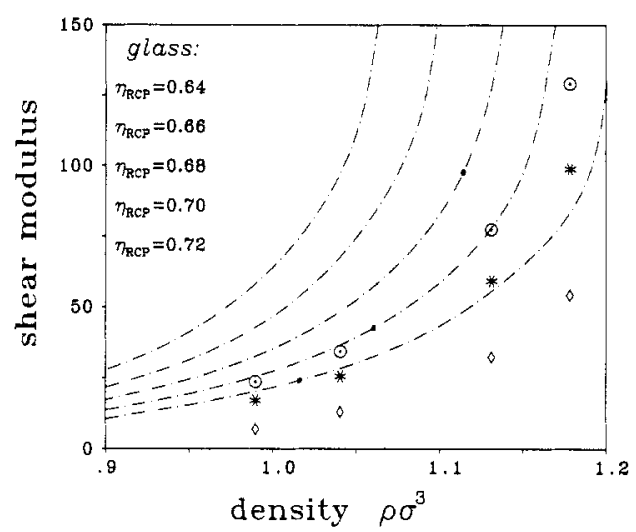

Figure 5. As figure 4 , but for the shear modulus $G$ (in units of $\left(\beta \sigma^{3}\right)^{-1}$ ). Also shown are FCC solid data: $C_{44}(\odot), \frac{1}{2}\left(C_{11}-C_{12}\right)(\diamond)$ and the Voigt estimate $G_{V}=\frac{1}{5}\left(C_{11}-C_{12}+3 C_{44}\right)(*)$ for a polycrystalline sample (from [22]).

$V$ being the undistorted volume. As is known from elastic properties of a crystal $[13,14]$, it is essential to take anisotropic Gaussian peaks for the local densities:

$\phi(\boldsymbol{r})=\left(\frac{\left(\alpha_{0}+\epsilon \alpha^{\prime}\right)\left(\alpha_{0}+\epsilon \tilde{\alpha}\right)^{2}}{\pi^{3}}\right)^{1 / 2} \exp \left(-\left(\alpha_{0}+\epsilon \alpha^{\prime}\right) x^{2}-\left(\alpha_{0}+\epsilon \tilde{\alpha}\right)\left(y^{2}+z^{2}\right)\right)$

where $\alpha_{0}$ is the known value for $\epsilon=0$ and $\tilde{\alpha}, \alpha^{\prime}$ are two additional variational parameters. If $C_{11}$ and $E$ are known, $G$ can readily be calculated. The results are illustrated in figure 5. Compared to the Voigt estimate [19] of an isotropic crystalline sample, the shear modulus of the glass is smaller if $\eta_{\mathrm{RCP}}$ and $\rho$ are high. Let us also remark that Poisson's ratio defined by

$$
\nu=\frac{C_{12}}{C_{11}+C_{12}}
$$

turns out to be positive for any $\rho$ and $\eta_{\mathrm{RCP}}$.

As already stated, this shows that, as far as the elastic response is concerned, the glass lies between liquid and solid and it also compares qualitatively well with computer simulations [15]. However, a final remark has to be added. On the base of simulations, it is commonly believed that the pressure is continuous but the elastic constants are discontinuous at the glass transition. In our approach, the glass transition is modelled quite simply by comparing the free energies of different density configurations. Therefore, it is first order, but in particular no dynamical effects are taken into account. The density functional approach, as it stands, is not suitable for studying the dynamics of the glass transition, but only for calculating the static properties of the glass itself.

\section{Final remarks and outlook}

First, one may worry about whether the Bennett structure is really a good description of the glass. In this context, it is interesting that this structure yields a glass form 
factor which agrees faily well with mode coupling theory, see [4] for details. On the other hand, it seems to be the simplest description where the site-site correlation function is explicitly known.

Second, it would be interesting to compare the elastic constants directly to the simulation data. As a first approximation one could use an effective diameter of the binary soft-sphere alloy of $[15,18]$ as an estimate for $\sigma$ and compare directly. This, however, is only a crude approximation. The difficulty is that molecular dynamics studies for a pure hard-sphere system yield a crystal but not a glass. This is one reason why an alloy was used in $[15,18]$.

In conclusion, the glass transition of the hard-sphere system was investigated using density functional theory and the elastic constants of the glass were calculated. In our case, it was found that the glass remains metastable with respect to the FCC crystal. It would be interesting to extend the density functional approach to the glass transition in more complex systems. First, a binary alloy may be investigated using density functional theory. For such a system, the site-site correlation function can be taken from simulation results, e.g. from [18]. By density functional theory, the free energy and the elastic constants of the glass can be calculated and can, in turn, be compared again with the simulation data.

A second interesting candidate is a polydisperse hard-sphere mixture which can be used modelling colloidal suspensions, see Pusey [20] for a recent review. For such a system, the stability of a crystal with respect to the fluid was already investigated [21] where it was found that the crystal is not stable for large polydispersities. It might be interesting to use a glassy density as input into the density functional for a polydisperse hard-sphere system and to check the stablity of the glass for high polydispersities. However, this is a harder task than to examine the crystal since the glassy site-site correlation functions are not known directly.

\section{Acknowledgments}

I thank J-P Hansen, for drawing my attention to this subject and for his kind hospitality at the Ecole Normale Supérieure de Lyon where this work was completed. Furthermore I am indebted to H Wagner, J N Roux, G Gompper and R Ohnesorge for helpful discussions.

\section{References}

[1] Woodcock L V and Angell C A 1981 Phys. Rev. Lett. 471129

[2] Singh Y, Stoessel J P and Wolynes P G 1985 Phys. Rev. Lett. 541059

[3] Baus M and Colot J L 1986 J. Phys. C: Solid State Phys. 19 L135

[4] Barrat J L, Götze W and Latz A 1989 J. Phys.: Condens. Matter 17163

[5] Ramakrishnan T V and Yussouf M 1979 Phys. Rev. B 192775

[6] Bennett C H 1972 J. Appl. Phys 432727

[7] Tarazona P 1985 Phys. Rev. A 312672

[8] Curtin W A and Ashcroft N W 1985 Phys. Rev. A 322909

[9] Denton A R and Ashcroft N W 1989 Phys. Rev. A 394701

[10] Lutsko J F and Baus M 1990 Phys. Rev. Lett. 64761

[11] Löwen H, Ohnesorge R and Wagner $\mathrm{H} 1990$ to be published

[12] Baus M 1990 J. Phys.: Condens. Matter 22111

[13] Velasco E and Tarazona P 1987 Phys. Rev. A 36979 
[14] Xu H and Baus M 1988 Phys. Rev. A 384348

[15] Barrat J L, Roux J N, Hansen J-P and Klein M L 1988 Europhys. Lett. 7707

[16] Kroll D M and Laird B B 1990 Phys. Rev. A to be published

[17] Baus $M$ and Colot J L 1985 Mol. Phys. 55653

[18] Roux J N, Barrat J L and Hansen J.P 1989 J. Phys.: Condens. Matter 17171 Roux J N 1990 to be published

[19] Hill R 1952 Proc. Phys. Soc. 65349

[20] Pusey P N 1990 Liguids, Freezing and the Glass Transition ed J-P Hansen, D Levesque and J Zinn-Justin (Amsterdam: North Holland) to be published

[21] Barrat J L and Hansen J-P $1986 \mathrm{~J}$. Physique 471547

[22] Frenkel D and Ladd A J C 1987 Phys. Rev. Lett. 591169 